\title{
Direct Contact with Platelets Induces Podoplanin Expression and Invasion in Human Oral Squamous Cell Carcinoma Cells
}

\author{
Se-Young Park ${ }^{1,2,+\dagger}$, Sun Kyoung Lee ${ }^{3, \dagger}$, Mihwa Lim ${ }^{3, \dagger}$, Bomi Kim ${ }^{3}$, Byeong-Oh Hwang ${ }^{1,2,3}$, Eunae Sandra Cho ${ }^{2,4,5}$, \\ Xianglan Zhang ${ }^{5,6}$, Kyung-Soo Chun ${ }^{7}$, Won-Yoon Chung ${ }^{1,2,3,5}$ and Na-Young Song ${ }^{1,2,3, *}$ \\ ${ }^{1}$ Department of Applied Life Science, The Graduate School, Yonsei University, Seoul 03722, \\ ${ }^{2}$ BK21 Four Project, Yonsei University College of Dentistry, Seoul 03722, \\ ${ }^{3}$ Department of Oral Biology, Yonsei University College of Dentistry, Seoul 03722, \\ ${ }^{4}$ Department of Oral Pathology, Yonsei University College of Dentistry, Seoul 03722, \\ ${ }^{5}$ Oral Cancer Research Institute, Yonsei University College of Dentistry, Seoul 03722, Republic of Korea \\ ${ }^{6}$ Department of Pathology, Yanbian University Hospital, Yanji City, Jilin Province 133000, China \\ ${ }^{7}$ College of Pharmacy, Keimyung University, Daegu 42601, Republic of Korea
}

\begin{abstract}
Oral squamous cell carcinoma (OSCC) is mostly diagnosed at an advanced stage, with local and/or distal metastasis. Thus, locoregional and/or local control of the primary tumor is crucial for a better prognosis in patients with OSCC. Platelets have long been considered major players in cancer metastasis. Traditional antiplatelet agents, such as aspirin, are thought to be potential chemotherapeutics, but they need to be used with caution because of the increased bleeding risk. Podoplanin (PDPN)-expressing cancer cells can activate platelets and promote OSCC metastasis. However, the reciprocal effect of platelets on PDPN expression in OSCC has not been investigated. In this study, we found that direct contact with platelets upregulated PDPN and integrin $\beta 1$ at the protein level and promoted invasiveness of human OSCC Ca9.22 cells that express low levels of PDPN. In another human OSCC HSC3 cell line that express PDPN at an abundant level, silencing of the PDPN gene reduced cell invasiveness. Analysis of the public database further supported the co-expression of PDPN and integrin $\beta 1$ and their increased expression in metastatic tissues compared to normal and tumor tissues of the oral cavity. Taken together, these data suggest that PDPN is a potential target to regulate platelet-tumor interaction and metastasis for OSCC treatment, which can overcome the limitations of traditional antiplatelet drugs.
\end{abstract}

Key Words: Oral cancer, Platelets, Podoplanin, Direct contact, Metastasis

\section{INTRODUCTION}

Oral squamous cell carcinoma (OSCC) is the most common type of head and neck squamous cell carcinoma (HNSCC), accounting for $3 \%$ of all cancer types and approximately $1.5 \%$ of cancer-related deaths in the United States (Chow, 2020). It is well known that patients with HNSCC with locally advanced features such as local invasion and lymph node metastasis often show relapse and thus, poor prognosis (Braakhuis et al., 2012). Therefore, understanding the locoregional characteristics of OSCC is important to identify prognostic markers and therapeutic targets.

Platelets are megakaryocyte-derived anucleated cells re- sponsible for maintaining hemostasis (Jurk and Kehrel, 2005). Although platelets lack genomic DNA, they secrete numerous granular constituents including serotonin, transforming growth factor- $\beta 1$, and platelet-derived growth factor, which regulate signal transduction in nearby cells (Flaumenhaft, 2003). Platelets can confer invasiveness and metastatic potential to cancer cells either via direct interactions or their releasates (Gay and Felding-Habermann, 2011; Labelle et al., 2011). During invasion, an initial stage in the metastatic cascade, cancer cells show dramatic changes in their plasticity and morphology, such as epithelial-to-mesenchymal transition (EMT) (van Zijl et al., 2011). Co-culturing with platelets induces the expression of EMT markers and enhances the invasiveness of

\section{Open Access https://doi.org/10.4062/biomolther.2021.167}

This is an Open Access article distributed under the terms of the Creative Commons Attribution Non-Commercial License (http://creativecommons.org/licenses/by-nc/4.0/) which permits unrestricted non-commercial use, distribution, and reproduction in any medium, provided the original work is properly cited.
Received Oct 25, 2021 Revised Dec 31, 2021 Accepted Jan 7, 2022

Published Online Feb 3, 2022

\section{*Corresponding Author}

E-mail: NYSONG608@yuhs.ac

Tel: +82-2-2228-3056, Fax: +82-2-364-7113

${ }^{\dagger}$ The first three authors contributed equally to this work. 
human and murine cancer cell lines (Wang et al., 2013; Guo et al., 2019). Moreover, platelet injection further promotes the formation of metastatic nodules in murine cancer metastasis models, suggesting that platelets play a crucial role in cancer cell invasion and metastasis (Haemmerle et al., 2017; Ichikawa et al., 2020). In line with these data, meta-analyses have revealed that higher platelet-to-lymphocyte ratios and/or platelet counts are significantly correlated with poorer overall survival (OS) in patients with HNSCC and OSCC (Rachidi et al., 2014; Bardash et al., 2019; Lee et al., 2020b; Wu et al., 2021).

Podoplanin (PDPN) is a transmembrane glycoprotein selectively expressed in lymphatic endothelial cells and renal podocytes (Astarita et al., 2012). PDPN is crucial for embryonic development and the lymphatic vascular system (Astarita et al., 2012). Cumulative evidence suggests that elevated PDPN expression, particularly at the invasive front, is associated with poor prognosis in various types of cancers, including OSCC (Yuan et al., 2006; Krishnan et al., 2018). Consistent with this data, PDPN has been reported to mediate cancer cell metastasis in vivo and in vitro. PDPN overexpression facilitates EMT marker expression and cell migration (Grau et al., 2015; Scholl et al., 2000). Notably, keratinocyte-specific ablation of PDPN leads to reduced tumor cell invasion in a two-stage skin carcinogenesis mouse model (Sesartic et al., 2020). These data suggest that PDPN is crucial for tumor invasion and metastasis.

Interestingly, platelets can interact with PDPN via its receptors, such as C-type lectin-like receptor 2 (CLEC2), and promote its metastatic potential (Lowe et al., 2012). Moreover, it has been reported that PDPN-positive OSCC cells induce platelet activation and aggregation in co-culture experiments (Lee et al., 2020a). However, whether platelets can regulate PDPN expression in OSCC cells has not yet been investigated. Here, we found that PDPN-positive oral tumors were concomitantly detected with CD42b-positive platelets in human OSCC tissues. Therefore, we sought to determine whether and how platelets regulate PDPN expression in OSCC cells.

\section{MATERIALS AND METHODS}

\section{Reagents}

The following antibodies were used for western blot analysis and immunohistochemical (IHC) staining: PDPN (sc-376695), integrin $\beta 1$ (ITGB1; sc-8978), and $\beta$-actin (sc-47778) from Santa Cruz Biotechnology (Santa Cruz, CA, USA); CD42b (ab183345) from Abcam (Cambridge, MA, USA); and PDPN (M3619) from Dako (Santa Clara, CA, USA).

\section{Hematoxylin and eosin (H\&E) and IHC staining}

Formalin-fixed paraffin-embedded (FFPE) sections of OSCC tissues were obtained from Yonsei University Dental Hospital (Seoul, Korea). All samples were approved by the Institutional Review Board of Yonsei University Dental Hospital (2-2021-0080). H\&E and IHC staining were performed on FFPE sections as previously described (Zhu et al., 2019).

\section{Public database analyses}

RNA sequencing (RNA-seq) and clinical data from The Cancer Genome Atlas (TCGA) cohorts were retrieved from the UCSC Xena browser (Goldman et al., 2020). To analyze pan-cancer cohorts, TCGA cohorts of 33 different cancer types were used to compare PDPN gene expression. For survival analysis, TCGA pan-cancer cohorts were stratified into PDPNhigh and -low groups or ITGB1-high and -low groups, based on median expression levels. Then, Kaplan-Meyer plots were generated using the UCSC Xena browser.

For HNSCC cohort analysis, genomic data commons (GDC) TCGA HNSC and/or HNSC (Puram, 2017) cohorts were utilized. HNSC cohorts were divided into tumor and non-tumor cells to compare PDPN and ITGB1 gene expression levels. Co-expression analysis was performed using the UCSC Xena browser.

Comparison of PDPN or ITGB1 expression between normal, tumor, and metastatic tissues was performed using gene chip data derived from the oral cavity using TNMplot (http:// tnmplot.com) (Bartha and Gyorffy, 2021).

\section{Platelet preparation}

Blood was collected from ICR mice (OrientBio, Gyeonggi, Korea) in a syringe filled with acid-citrate-dextrose buffer (Sigma-Aldrich, St. Louis, MO, USA) by cardiac puncture. Platelets were prepared as previously described (Aurbach et al., 2019). Briefly, pooled murine blood was centrifuged at $180 \times \mathrm{g}$ for $10 \mathrm{~min}$. The platelet-rich plasma at the bottom was then rinsed three times with wash buffer and centrifuged at $1,800 \times \mathrm{g}$ for $10 \mathrm{~min}$. Platelet pellets were resuspended at the desired density. All animal procedures were approved by the Institutional Animal Care and Use Committee (IACUC) of the Yonsei University (Seoul, Korea) (2019-0292).

\section{Cell culture and co-culture with platelets}

Cells were cultured in Dulbecco's modified Eagle's medium (DMEM) containing 10\% (v/v) fetal bovine serum and antibiotics. For direct co-culture, Ca9.22 cells were seeded in 100 $\mathrm{mm}$ dishes at $2 \times 10^{6}$ cells per well. The next day, the platelet suspension $\left(5 \times 10^{6}\right.$ cells per well) was directly added to the growing cells. After $24 \mathrm{~h}$ of direct co-culture, the conditioned medium (CM) was collected and centrifuged at $1,800 \times \mathrm{g}$ for 10 min to remove platelets. Another batch of $\mathrm{Ca9} .22$ cells was then treated with $70 \% \mathrm{CM}$ for $24 \mathrm{~h}$.

\section{Western blot analysis}

Cell lysates were loaded on an acrylamide gel, and protein expression levels were determined by western blot analysis as described previously (Song et al., 2018). Briefly, cell lysates $(10-20 \mu \mathrm{g})$ were suspended in a protein gel loading buffer (Bio-Rad Laboratories, Hercules, CA, USA), heated at $95^{\circ} \mathrm{C}$ for $5 \mathrm{~min}$, and then resolved using $7 \%-12 \%$ sodium dodecyl sulfate polyacrylamide gel electrophoresis (SDS-PAGE). The separated proteins were transferred to polyvinylidene fluoride membranes and analyzed using the indicated antibodies. Chemiluminescence reactions were performed using an HRP substrate (Amersham, GE Healthcare, Little Chalfont, UK), and the signal was detected and recorded using an image analyzer (Amersham).

\section{Invasion assay}

Transwell invasion assay was performed using Matrigelcoated inserts (Corning Costar, Cambridge, MA, USA). Ca9.22 cells $\left(1 \times 10^{3}\right.$ per well) were placed in the insert $2 \mathrm{~h}$ prior to the addition of platelets $\left(1 \times 10^{5}\right.$ per well). Ca9.22 cells in contact with or without platelets were allowed to invade for 
$24 \mathrm{~h}$. The invasive cells were stained with Mayer's hematoxylin (Cancer Diagnostics Inc., Durham, NC, USA) and counted under a light microscope.

\section{siRNA-mediated knockdown of PDPN and invasion assay}

HSC3 cells were transfected with scrambled or PDPN siRNAs (Origene Technologies, Rockville, MD, USA) using Lipofectamine $^{\mathrm{TM}}$ RNAi-MAX transfection reagent (Invitrogen, Carlsbad, CA, USA) according to the manufacturer's instructions. The cells were then placed in a Matrigel-coated insert (Corning Costar) for the transwell invasion assay. HSC3 cells transfected with scrambled or PDPN siRNA were allowed to invade for $24 \mathrm{~h}$. The invasive cells were stained with Mayer's hematoxylin (Cancer Diagnostics Inc.) and counted under a light microscope.

\section{Statistical analysis}

Statistical analyses were performed using the GraphPad Prism software (GraphPad Software, San Diego, CA, USA). Pearson and Spearman analyses were used to determine the correlation between the two different gene expression levels. Student's $t$-test was used for comparisons between groups. Statistical significance between groups was determined using the Kruskal-Wallis's test, Welch's test, or one-way analysis of variance (ANOVA). Kaplan-Meier analysis and log-rank test were used for survival analysis. All experiments were performed in triplicate and the results are presented as the mean \pm standard deviation (SD). In all tests, statistical significance was set at $p<0.05$.

\section{RESULTS}

\section{PDPN-positive tumors and CD42b-positive platelets are detected in human oral cancer tissues}

First, we examined PDPN expression profile in various types of cancers in the TCGA pan-cancer cohort. Among the 33 cancer types, HNSCC presented relatively higher PDPN expression than the other types of cancers (top 4, Supplementary Fig. 1). Moreover, PDPN expression was significantly

A
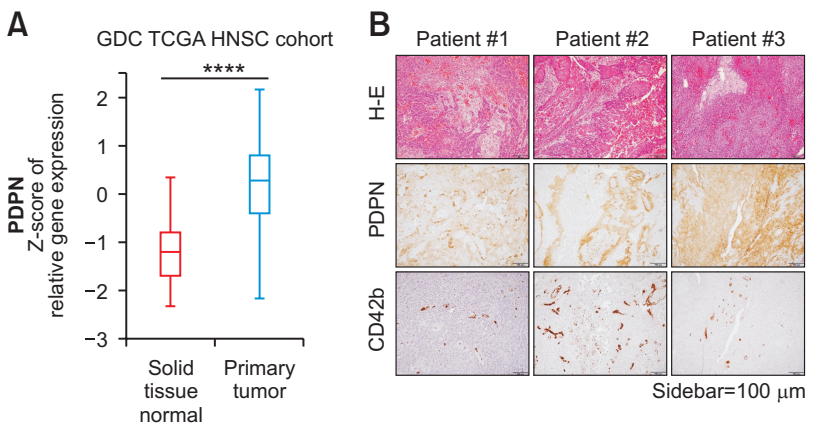

Fig. 1. Co-expression of PDPN and CD42b in human OSCC tumor sections. (A) UCSC Xena browser-based comparative analysis of PDPN gene expression in normal solid tissue (red) and primary tumor (blue) samples in GDC TCGA HNSC cohort. Statistical significance was assessed using one-way ANOVA. ${ }^{* * * *} p<0.0001$. (B) H\&E (top) and IHC staining of PDPN (middle) and CD42b (bottom) in human OSCC tissues from three different patients. Representative images are shown. elevated in tumor tissues compared to that in normal tissues in the HNSCC cohort (Fig. 1A). These data support a strong association between PDPN expression and OSCC.

Lee et al. (2020a) demonstrated that PDPN-positive tumors contain more platelets than PDPN-low or PDPN-negative tumors in a xenograft mouse model. Therefore, we examined the relationship between PDPN expression and platelets in human OSCC tissues. An antibody against CD42b, a platelet surface membrane glycoprotein, was used to identify the platelets (Ishikawa et al., 2016). Tumor cells stained positively for PDPN in human OSCC tissues (Fig. 1B). CD42b-positive platelets were also detected in human OSCC tissue sections and were found to particularly aggregate within blood vessels (Fig. 1B). IHC analyses indicated that intravascular platelet aggregation was concomitant with PDPN-positive OSCC tumors.

\section{Platelets promote PDPN expression and cell invasion via direct contact in Ca9.22 cells}

PDPN mediates tumor-induced platelet activation (SuzukiInoue et al., 2007; Ward et al., 2019). Conversely, we investigated whether platelets induced PDPN expression in OSCC cells. Based on screening of PDPN expression in different human OSCC cell lines, Ca9.22 cells were selected because of their low level of PDPN expression (Supplementary Fig. 2). Two different culture methods were adopted to distinguish between the direct and indirect effects of platelets on OSCC cells. As illustrated in Fig. 2A, Ca9.22 cells were directly cocultured with platelets or incubated with platelet releasatecontaining $\mathrm{CM}$. Direct co-culture with platelets markedly upregulated PDPN expression, which was not altered by $\mathrm{CM}$ treatment (Fig. 2B, 2C). These data suggest that platelets promote PDPN overexpression in human OSCC Ca9.22 cells via physical contact rather than by their releasates.

Because PDPN can induce invasion of OSCCs (Hwang et al., 2012; Retzbach et al., 2018), we examined whether direct contact with platelets can regulate the invasiveness of Ca9.22 cells. Indeed, the number of invading cells was significantly increased by physical contact with platelets, suggesting that physical interaction with platelets is crucial for the induction of PDPN expression and invasiveness of Ca9.22 cells (Fig. 2D).

\section{PDPN correlates with ITGB1 expression and promotes invasion in human OSCC}

Next, we investigated whether direct contact with platelets or platelet releasates affects the expression levels of proteins responsible for cell invasion and EMT. Neither direct co-culture nor CM treatment altered the expression of EMT markers such as E-cadherin, $\mathrm{N}$-cadherin, and vimentin (data not shown). However, we observed that direct contact with platelets led to upregulation of ITGB1 and PDPN in Ca9.22 cells (Fig. 2B, 2C). Elevated levels of ITGB1 and PDPN are associated with increased metastatic potential in patients with HNSCC and/ or OSCC (Hwang et al., 2012; Tsuneki et al., 2013; Lin et al., 2014; Shrivastava et al., 2015). Similar to PDPN expression (Fig. 1A), HNSCC cohort analysis showed that ITGB1 expression levels were markedly higher in tumor tissues than in normal tissues (Supplementary Fig. 3A). Moreover, PDPN expression was positively correlated with ITGB1 expression in the two HNSCC datasets (Fig. 3A, Supplementary Fig. 3B). It is well documented that ITGB1 contributes to EMT and invasion of cancer cells (Bhowmick et al., 2001; Shibue and Wein- 
A

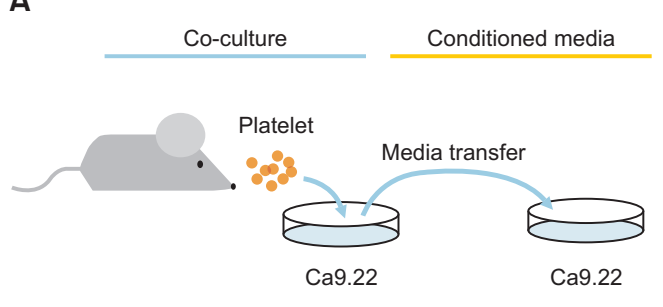

B

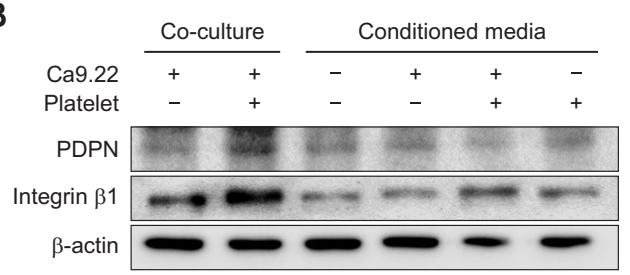

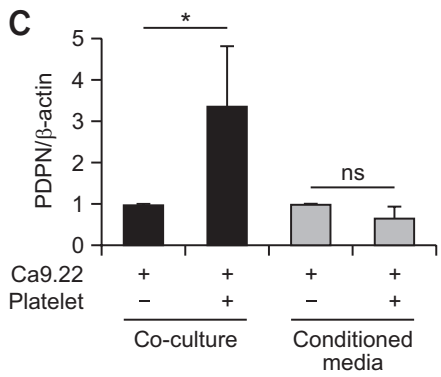

D
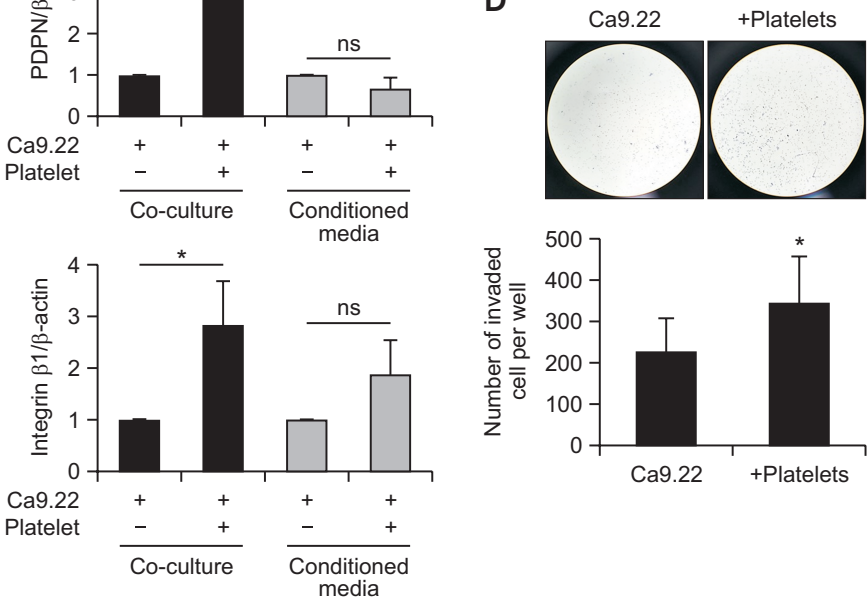

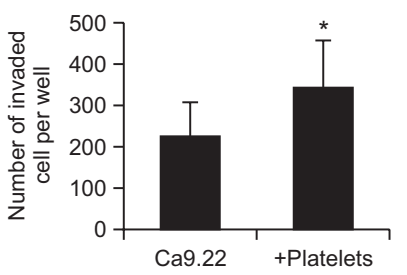

Ca9.22+Platelets

Fig. 2. Direct contact with platelets induces PDPN expression and invasiveness in Ca9.22 cells. (A) Experimental scheme. For direct coculture, platelets isolated from ICR mice were directly added to Ca9.22 cells. After incubation for $24 \mathrm{~h}$, the culture medium was transferred to newly prepared Ca9.22 cells. (B, C) Immunoblot analysis (B) of PDPN and integrin $\beta 1$ (ITGB1) expression in Ca9.22 cells co-cultured with platelets or incubated with conditioned medium as indicated. $\beta$-actin was used as a loading control for immunoblotting. Band intensities were normalized to the actin level and are presented as bar graphs (C). ns, not significant; ${ }^{*} p<0.05$. (D) Ca9.22 cells were seeded in the insert $2 \mathrm{~h}$ prior to addition of platelets, and then allowed to invade for $24 \mathrm{~h}$. The invaded cells were stained and counted. The bar graph presents the quantitative data of the invasion assay (top). ${ }^{*} p<0.05$. Representative images are displayed (bottom).

A

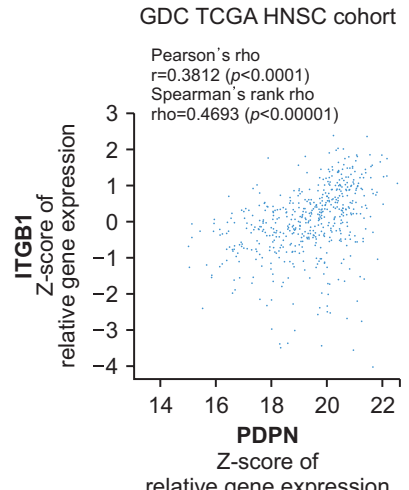

B
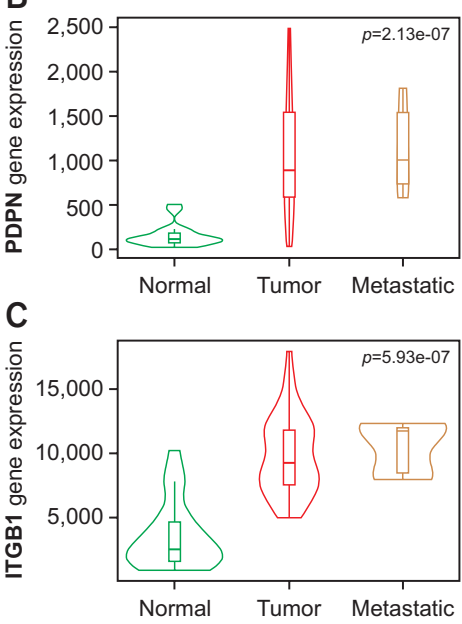
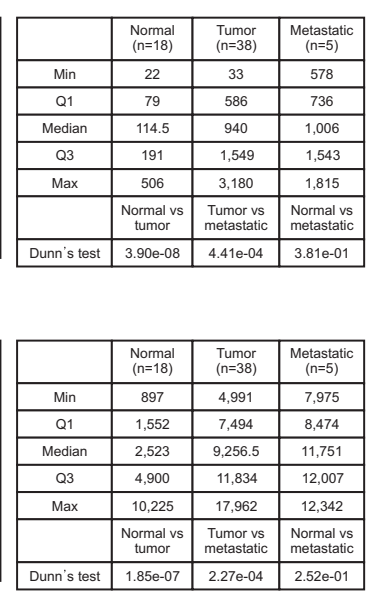

D
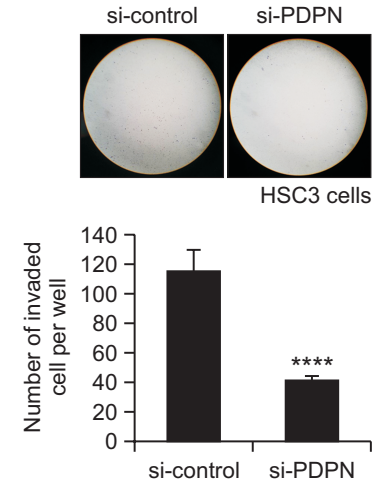

Fig. 3. Correlation between PDPN and ITGB1 expression and their involvement in metastasis. (A) Co-expression analyses of PDPN and ITGB1 were performed in the GDC TCGA HNSC cohort using the UCSC Xena browser. (B, C) Violin plots of PDPN (B) and ITGB1 (C) gene expression in the oral cancer comparing normal (green), tumor (red), and metastatic (yellow) tissues from the gene chip data at TNMplot. com. Statistical significance was determined using Kruskal-Wallis and Dunn's post hoc tests. (D) HSC3 cells transfected with si-control or si-PDPN $(20 \mathrm{nM})$ for $48 \mathrm{~h}$ were seeded in the insert, and then allowed to invade for $24 \mathrm{~h}$. The invaded cells were stained and counted. The bar graph presents the quantitative data of the invasion assay (top). ${ }^{* * *} p<0.0001$. Representative images are displayed (bottom).

berg, 2009; Cooper and Giancotti, 2019). In line with these data, TNM plot analysis using gene chip data revealed that gene expression of PDPN (Fig. 3B) and ITGB1 (Fig. 3C) was higher in metastatic tissues than in normal and tumor tissues in oral cancer. Taken together, these data suggest that the co-expression of PDPN and ITGB1 is closely associated with metastasis in OSCC.

To validate the metastatic potential of PDPN in OSCC, we used HSC3 cells that express high levels of PDPN compared to Ca9.22 cells with low PDPN expression (Supplementary Fig. 2). Knockdown of PDPN reduced the invasiveness of HSC3 cells (Fig. 3D, Supplementary Fig. 3C), implying that platelet-induced PDPN expression promotes metastatic potential in human OSCC. 
A

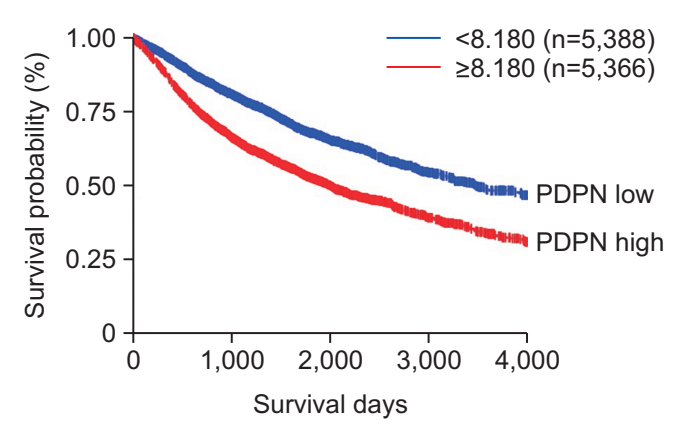

B

$$
\text { TCGA pan cancer cohort }
$$
Log-rank $p<0.0001$

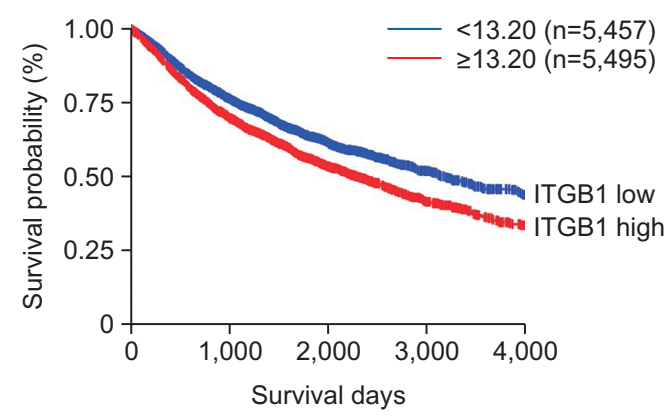

Fig. 4. Prognostic significance of PDPN and ITGB1 expression. (A, B) Kaplan-Meier plots showing the overall survival (OS) of the pan-cancer cohorts in TCGA dataset. The OS group stratified by PDPN-high and -low patient groups (A); The OS group stratified by ITGB1-high and -low patient groups (B).

\section{Overexpression of PDPN and ITGB1 is associated with poor prognosis in pan-cancer cohort}

We then examined the prognostic values of PDPN and ITGB1 by analyzing OS in TCGA datasets. In TCGA tumor samples spanning 33 cancer types, higher expression of $P D P N$ or ITGB1 was strongly associated with poorer OS (Fig. $4 A, 4 B$ ). Although we did not observe any difference in OS based on the expression levels of PDPN or ITGB1 in the two HNSCC cohorts (data not shown), Retzbach et al. (2018) reported that PDPN expression was inversely associated with OS in oral cancer patients. Taken together these data suggest that PDPN and ITGB1 are markers of poor prognosis in cancer patients, regardless of the cancer type.

\section{DISCUSSION}

Early detection of cancer is the most important factor in improving patient survival rates. The entire mouth is highly accessible for visual examination to screen for oral cancers. However, most OSCC cases are not detected at the early stage, but rather diagnosed at the advanced stages with lymph node and distant metastasis (Bugshan and Farooq, 2020). Distant metastasis from OSCC is relatively rare but strongly correlated with poor prognosis (Irani, 2016). Thus, successful locoregional control of the primary tumor is a key factor in improving the outcome and survival rate of patients with OSCC.

A large body of evidence supports that an increased platelet count is strongly associated with worse prognosis in many types of cancers, including OSCC (Ikeda et al., 2002; Yu et al., 2013; Rachidi et al., 2014). Platelets directly interact with tumors and promote cancer metastasis (Gay and FeldingHabermann, 2011; Labelle et al., 2011, 2014). Co-incubation with platelets increases invasive mesenchymal-like phenotypes and metastatic potential in mouse breast and colon cancer cell lines (Labelle et al., 2011). However, PDPN deficiency downregulated platelet-induced expression of EMT markers in human esophageal squamous carcinoma cells, suggesting that PDPN is crucial for cancer metastasis induced by tumorplatelet interaction (Watanabe et al., 2020). In this study, we found that direct contact with platelets promotes PDPN expression and cell invasion in human OSCC cells. Consistent with previous reports, PDPN silencing was found to reduce the motility of human oral cancer cells.

We found that PDPN expression highly correlated with that of ITGB1, which is involved in EMT. It has been reported that highly metastatic OSCC cells exhibit elevated expression of ITGB1 compared to less metastatic cells (Zhang et al., 2002). Moreover, ITGB1-high oral epidermoid carcinoma cells were more tumorigenic in a xenograft model than in ITGB1-low cells (Lin et al., 2014). Taken together, these results suggest that platelets can promote cancer metastasis through upregulation of PDPN and ITGB1. Notably, ITGB1 expression was slightly enhanced following CM treatment, whereas PDPN upregulation was solely mediated by direct co-culture with platelets. Thus, PDPN expression is highly dependent on direct contact with platelets in OSCC cells. Together with a previous report on platelet activation by PDPN-positive OSCC cells, these data suggest that this bidirectional communication between OSCC cells and platelets may be a promising therapeutic target in OSCC treatment, particularly through locoregional control of OSCC (Lee et al., 2020a).

In line with this notion, antiplatelet drug intake was associated with prolonged survival in patients with HNSCC, suggesting that antiplatelet medicines may serve as potential chemotherapeutics (Rachidi et al., 2014; Furlan et al., 2015). However, traditional antiplatelet agents, including aspirin, are known to increase the risk of bleeding, which limits their potential application as anticancer drugs (Serebruany et al., 2015; Wojtukiewicz et al., 2017). Thus, it is necessary to identify new molecular targets that inhibit platelet-cancer interaction without causing unfavorable effects, such as hemorrhage. It has been reported that cobalt hematoporphyrin inhibits lung metastasis of PDPN-expressing murine melanoma cells via blocking interaction between PDPN and platelet CLEC2 (Tsukiji et al., 2018). Based on these investigations, targeting PDPN may be a better approach for OSCC treatment compared to traditional antiplatelet agents.

\section{CONFLICT OF INTEREST}

The authors declare that they have no known competing financial interests or personal relationships that could have influenced the work reported in this study. 


\section{ACKNOWLEDGMENTS}

This study was supported by Yonsei University College of Dentistry Fund (6-2019-0022).

\section{REFERENCES}

Astarita, J. L., Acton, S. E. and Turley, S. J. (2012) Podoplanin: emerging functions in development, the immune system, and cancer. Front. Immunol. 3, 283.

Aurbach, K., Spindler, M., Haining, E. J., Bender, M. and Pleines, I. (2019) Blood collection, platelet isolation and measurement of platelet count and size in mice-a practical guide. Platelets 30, 698707.

Bardash, Y., Olson, C., Herman, W., Khaymovich, J., Costantino, P. and Tham, T. (2019) Platelet-lymphocyte ratio as a predictor of prognosis in head and neck cancer: a systematic review and metaanalysis. Oncol. Res. Treat. 42, 665-677.

Bartha, A. and Gyorffy, B. (2021) TNMplot.com: a web tool for the comparison of gene expression in normal, tumor and metastatic tissues. Int. J. Mol. Sci. 22, 2622.

Bhowmick, N. A., Zent, R., Ghiassi, M., McDonnell, M. and Moses, H. L. (2001) Integrin beta 1 signaling is necessary for transforming growth factor-beta activation of p38MAPK and epithelial plasticity. J. Biol. Chem. 276, 46707-46713.

Braakhuis, B. J., Brakenhoff, R. H. and Leemans, C. R. (2012) Treatment choice for locally advanced head and neck cancers on the basis of risk factors: biological risk factors. Ann. Oncol. 23 Suppl 10, x173-x177.

Bugshan, A. and Farooq, I. (2020) Oral squamous cell carcinoma: metastasis, potentially associated malignant disorders, etiology and recent advancements in diagnosis. F1000Res. 9, 229.

Chow, L. Q. M. (2020) Head and neck cancer. N. Engl. J. Med. 382 60-72.

Cooper, J. and Giancotti, F. G. (2019) Integrin signaling in cancer: mechanotransduction, stemness, epithelial plasticity, and therapeutic resistance. Cancer Cell 35, 347-367.

Flaumenhaft, R. (2003) Molecular basis of platelet granule secretion. Arterioscler. Thromb. Vasc. Biol. 23, 1152-1160.

Furlan, C., Steffan, A., Polesel, J., Trovo, M., Gobitti, C., Vaccher, E., Serraino, D., Barzan, L. and Franchin, G. (2015) Lower platelet counts and antiplatelet therapy independently predict better outcomes in patients with head and neck squamous cell carcinoma: a retrospective analysis. Biomark. Res. 3, 25.

Gay, L. J. and Felding-Habermann, B. (2011) Platelets alter tumor cell attributes to propel metastasis: programming in transit. Cancer Cell 20, 553-554.

Goldman, M. J., Craft, B., Hastie, M., Repečka, K., McDade, F., Kamath, A., Banerjee, A., Luo, Y., Rogers, D., Brooks, A. N., Zhu, J. and Haussler, D. (2020) Visualizing and interpreting cancer genomics data via the Xena platform. Nat. Biotechnol. 38, 675-678.

Grau, S. J., Trillsch, F., Tonn, J. C., Goldbrunner, R. H., Noessner, E., Nelson, P. J. and von Luettichau, I. (2015) Podoplanin increases migration and angiogenesis in malignant glioma. Int. J. Clin. Exp. Pathol. 8, 8663-8670.

Guo, Y., Cui, W., Pei, Y. and Xu, D. (2019) Platelets promote invasion and induce epithelial to mesenchymal transition in ovarian cancer cells by TGF-beta signaling pathway. Gynecol. Oncol. 153, 639650.

Haemmerle, M., Taylor, M. L., Gutschner, T., Pradeep, S., Cho, M. S., Sheng, J., Lyons, Y. M., Nagaraja, A. S., Dood, R. L., Wen, Y., Mangala, L. S., Hansen, J. M., Rupaimoole, R., Gharpure, K. M., Rodriguez-Aguayo, C., Yim, S. Y., Lee, J. S., Ivan, C., Hu, W., Lopez-Berestein, G., Wong, S. T., Karlan, B. Y., Levine, D. A., Liu, J., Afshar-Kharghan, V. and Sood, A. K. (2017) Platelets reduce anoikis and promote metastasis by activating YAP1 signaling. Nat Commun. 8, 310

Hwang, Y. S., Xianglan, Z., Park, K. K. and Chung, W. Y. (2012) Functional invadopodia formation through stabilization of the PDPN transcript by IMP-3 and cancer-stromal crosstalk for PDPN expres- sion. Carcinogenesis 33, 2135-2146

Ichikawa, J., Ando, T., Kawasaki, T., Sasaki, T., Shirai, T., Tsukiji, N. Kimura, Y., Aoki, K., Hayakawa, K., Suzuki-Inoue, K., Saitoh, M. and Haro, H. (2020) Role of platelet C-type lectin-like receptor 2 in promoting lung metastasis in osteosarcoma. J. Bone Miner. Res. 35, 1738-1750.

Ikeda, M., Furukawa, H., Imamura, H., Shimizu, J., Ishida, H., Masutani, S., Tatsuta, M. and Satomi, T. (2002) Poor prognosis associated with thrombocytosis in patients with gastric cancer. Ann. Surg. Oncol. 9, 287-291.

Irani, S. (2016) Distant metastasis from oral cancer: a review and molecular biologic aspects. J. Int. Soc. Prev. Community Dent. 6, 265-271.

Ishikawa, S., Miyashita, T., Inokuchi, M., Hayashi, H., Oyama, K., Tajima, H., Takamura, H., Ninomiya, I., Ahmed, A. K., Harman, J. W., Fushida, S. and Ohta, T. (2016) Platelets surrounding primary tumor cells are related to chemoresistance. Oncol. Rep. 36, 787794.

Jurk, K. and Kehrel, B. E. (2005) Platelets: physiology and biochemistry. Semin. Thromb. Hemost. 31, 381-392.

Krishnan, H., Rayes, J., Miyashita, T., Ishii, G., Retzbach, E. P., Sheehan, S. A., Takemoto, A., Chang, Y. W., Yoneda, K., Asai, J., Jensen, L., Chalise, L., Natsume, A. and Goldberg, G. S. (2018) Podoplanin: an emerging cancer biomarker and therapeutic target. Cancer Sci. 109, 1292-1299.

Labelle, M., Begum, S. and Hynes, R. O. (2011) Direct signaling between platelets and cancer cells induces an epithelial-mesenchymal-like transition and promotes metastasis. Cancer Cell 20, 576590.

Labelle, M., Begum, S. and Hynes, R. O. (2014) Platelets guide the formation of early metastatic niches. Proc. Natl. Acad. Sci. U.S.A. 111, E3053- E3061

Lee, H. Y., Yu, N. Y., Lee, S. H., Tsai, H. J., Wu, C. C., Cheng, J. C., Chen, D. P., Wang, Y. R. and Tseng, C. P. (2020a) Podoplanin promotes cancer-associated thrombosis and contributes to the unfavorable overall survival in an ectopic xenograft mouse model of oral cancer. Biomed. J. 43, 146-162.

Lee, S., Kim, D. W., Kwon, S., Kim, H. J., Cha, I. H. and Nam, W. (2020b) Prognostic value of systemic inflammatory markers for oral cancer patients based on the 8th edition of AJCC staging system. Sci. Rep. 10, 12111.

Lin, H. C., Wu, C. L., Chen, Y. L., Huang, J. S., Wong, T. Y. and Yuan, K. (2014) High-level beta1-integrin expression in a subpopulation of highly tumorigenic oral cancer cells. Clin. Oral Investig. 18, 1277-1284

Lowe, K. L., Navarro-Nunez, L. and Watson, S. P. (2012) Platelet CLEC-2 and podoplanin in cancer metastasis. Thromb. Res. 129 Suppl 1, S30-S37.

Puram, S. V., Tirosh, I., Parikh, A. S., Patel, A. P., Yizhak, K., Gillespie, S., Rodman, C., Luo, C. L., Mroz, E. A., Emerick, K. S., Deschler, D. G., Varvares, M. A., Mylvaganam, R., Rozenblatt-Rosen, O., Rocco, J. W., Faquin, W. C., Lin, D. T., Regev, A. and Bernstein, B. E. (2017) Single-cell transcriptomic analysis of primary and metastatic tumor ecosystems in head and neck cancer. Cell 171, 16111624.e24.

Rachidi, S., Wallace, K., Day, T. A., Alberg, A. J. and Li, Z. (2014) Lower circulating platelet counts and antiplatelet therapy independently predict better outcomes in patients with head and neck squamous cell carcinoma. J. Hematol. Oncol. 7, 65.

Retzbach, E. P., Sheehan, S. A., Nevel, E. M., Batra, A., Phi, T., Nguyen, A. T. P., Kato, Y., Baredes, S., Fatahzadeh, M., Shienbaum, A. J. and Goldberg, G. S. (2018) Podoplanin emerges as a functionally relevant oral cancer biomarker and therapeutic target. Oral Oncol. 78, 126-136.

Scholl, F. G., Gamallo, C. and Quintanilla, M. (2000) Ectopic expression of PA2.26 antigen in epidermal keratinocytes leads to destabilization of adherens junctions and malignant progression. Lab. Invest. 80, 1749-1759.

Serebruany, V. L., Cherepanov, V., Cabrera-Fuentes, H. A. and Kim, M. H. (2015) Solid cancers after antiplatelet therapy: confirmations, controversies, and challenges. Thromb. Haemost. 114, 1104-1112.

Sesartic, M., Ikenberg, K., Yoon, S. Y. and Detmar, M. (2020) Kera- 
tinocyte-expressed podoplanin is dispensable for multi-step skin carcinogenesis. Cells 9, 1542.

Shibue, T. and Weinberg, R. A. (2009) Integrin beta1-focal adhesion kinase signaling directs the proliferation of metastatic cancer cells disseminated in the lungs. Proc. Natl. Acad. Sci. U.S.A. 106, 10290-10295

Shrivastava, S., Steele, R., Sowadski, M., Crawford, S. E., Varvares, M. and Ray, R. B. (2015) Identification of molecular signature of head and neck cancer stem-like cells. Sci. Rep. 5, 7819.

Song, N. Y., Zhu, F., Wang, Z., Willette-Brown, J., Xi, S., Sun, Z., Su, L., Wu, X., Ma, B., Nussinov, R., Xia, X., Schrump, D. S., Johnson, P. F., Karin, M. and Hu, Y. (2018) IKKalpha inactivation promotes Kras-initiated lung adenocarcinoma development through disrupting major redox regulatory pathways. Proc. Natl. Acad. Sci. U.S.A. 115, E812-E821.

Suzuki-Inoue, K., Kato, Y., Inoue, O., Kaneko, M. K., Mishima, K., Yatomi, Y., Yamazaki, Y., Narimatsu, H. and Ozaki, Y. (2007) Involvement of the snake toxin receptor CLEC-2, in podoplanin-mediated platelet activation, by cancer cells. J. Biol. Chem. 282, 2599326001.

Tsukiji, N., Osada, M., Sasaki, T., Shirai, T., Satoh, K., Inoue, O., Umetani, N., Mochizuki, C., Saito, T., Kojima, S., Shinmori, H., Ozaki, Y. and Suzuki-Inoue, K. (2018) Cobalt hematoporphyrin inhibits CLEC-2-podoplanin interaction, tumor metastasis, and arte$\mathrm{rial} / \mathrm{venous}$ thrombosis in mice. Blood Adv. 2, 2214-2225.

Tsuneki, M., Yamazaki, M., Maruyama, S., Cheng, J. and Saku, T. (2013) Podoplanin-mediated cell adhesion through extracellular matrix in oral squamous cell carcinoma. Lab. Invest. 93, 921-932.

van Zijl, F., Krupitza, G. and Mikulits, W. (2011) Initial steps of metastasis: cell invasion and endothelial transmigration. Mutat. Res. 728, 23-34.

Wang, Y., Sun, Y., Li, D., Zhang, L., Wang, K., Zuo, Y., Gartner, T. K. and Liu, J. (2013) Platelet P2Y12 is involved in murine pulmonary metastasis. PLOS ONE 8, e80780.
Ward, L. S. C., Sheriff, L., Marshall, J. L., Manning, J. E., Brill, A., Nash, G. B. and McGettrick, H. M. (2019) Podoplanin regulates the migration of mesenchymal stromal cells and their interaction with platelets. J. Cell Sci. 132, jcs222067.

Watanabe, N., Kidokoro, M., Tanaka, M., Inoue, S., Tsuji, T., Akatuska, H., Okada, C., lida, Y., Okada, Y., Suzuki, Y., Sato, T., Yahata, T., Hirayama, N., Nakagawa, Y. and Inokuchi, S. (2020) Podoplanin is indispensable for cell motility and platelet-induced epithelial-tomesenchymal transition-related gene expression in esophagus squamous carcinoma TE11A cells. Cancer Cell Int. 20, 263.

Wojtukiewicz, M. Z., Hempel, D., Sierko, E., Tucker, S. C. and Honn, K. V. (2017) Antiplatelet agents for cancer treatment: a real perspective or just an echo from the past? Cancer Metastasis Rev. 36, 305-329.

Wu, Y. Y., Chang, K. P., Ho, T. Y., Chou, W. C., Hung, S. P., Fan, K. H., Chiang, Y. Y., Chou, Y. C. and Tsang, N. M. (2021) Comparative prognostic value of different preoperative complete blood count cell ratios in patients with oral cavity cancer treated with surgery and postoperative radiotherapy. Cancer Med. 10, 1975-1988.

Yu, D., Liu, B., Zhang, L. and Du, K. (2013) Platelet count predicts prognosis in operable non-small cell lung cancer. Exp. Ther. Med. 5, 1351-1354.

Yuan, P., Temam, S., El-Naggar, A., Zhou, X., Liu, D. D., Lee, J. J. and Mao, L. (2006) Overexpression of podoplanin in oral cancer and its association with poor clinical outcome. Cancer 107, 563-569.

Zhang, X., Liu, Y., Gilcrease, M. Z., Yuan, X. H., Clayman, G. L., AdlerStorthz, K. and Chen, Z. (2002) A lymph node metastatic mouse model reveals alterations of metastasis-related gene expression in metastatic human oral carcinoma sublines selected from a poorly metastatic parental cell line. Cancer 95, 1663-1672.

Zhu, L., Cho, E., Zhao, G., Roh, M. R. and Zheng, Z. (2019) The pathogenic effect of cortactin tyrosine phosphorylation in cutaneous squamous cell carcinoma. In Vivo 33, 393-400. 